\title{
Response surface modeling of processing parameters for the preparation of phytosterol Nanodispersions using an emulsification-evaporation technique.
}

\begin{abstract}
The purpose of this study was to optimize the production parameters for water-soluble phytosterol nanodispersions. Response surface methodology (RSM) was employed to model and optimize three of the processing parameters: mixing time $(\mathrm{t})$ by conventional homogenizer (1-20 min), mixing speed (v) by conventional homogenizer (1,000-9,000 rpm) and homogenization pressure $(\mathrm{P})$ by high-pressure homogenizer $(0.1-80 \mathrm{MPa})$. All responses [i.e., mean particle size (PS), polydispersity index (PDI) and phytosterols concentration (Phyto, mg/l)] fitted well to a reduced quadratic model by multiple regressions after manual elimination. For PS, PDI and Phyto, the coefficients of determination (R 2) were $0.9902,0.9065$ and 0.8878 , respectively. The optimized processing parameters were 15.25 min mixing time, 7,000 rpm mixing speed and homogenization pressure $42.4 \mathrm{MPa}$. In the produced nanodispersions, the corresponding responses for the optimized preparation conditions were a PS of $52 \mathrm{~nm}$, PDI of 0.3390 and a Phyto of $336 \mathrm{mg} / \mathrm{l}$.
\end{abstract}

Keyword: Phytosterol; Nanodispersion; Response surface methodology; High-pressure. 